The University of Maine

DigitalCommons@UMaine

Marine Sciences Faculty Scholarship

School of Marine Sciences

$2-20-2002$

\title{
Phase function effects on oceanic light fields
}

Curtis D. Mobley

Sequoia Scientific, Inc.

Lydia K. Sundman

Sequoia Scientific, Inc.

Emmanuel Boss

Oregon State University, emmanuel.boss@maine.edu

Follow this and additional works at: https://digitalcommons.library.umaine.edu/sms_facpub

Part of the Marine Biology Commons

\section{Repository Citation}

Mobley, Curtis D.; Sundman, Lydia K.; and Boss, Emmanuel, "Phase function effects on oceanic light fields" (2002). Marine Sciences Faculty Scholarship. 146.

https://digitalcommons.library.umaine.edu/sms_facpub/146

This Article is brought to you for free and open access by DigitalCommons@UMaine. It has been accepted for inclusion in Marine Sciences Faculty Scholarship by an authorized administrator of DigitalCommons@UMaine. For more information, please contact um.library.technical.services@maine.edu. 


\title{
Phase function effects on oceanic light fields
}

\author{
Curtis D. Mobley, Lydia K. Sundman, and Emmanuel Boss
}

\begin{abstract}
Numerical simulations show that underwater radiances, irradiances, and reflectances are sensitive to the shape of the scattering phase function at intermediate and large scattering angles, although the exact shape of the phase function in the backscatter directions (for a given backscatter fraction) is not critical if errors of the order of $10 \%$ are acceptable. We present an algorithm for generating depth- and wavelength-dependent Fournier-Forand phase functions having any desired backscatter fraction. Modeling of a comprehensive data set of measured inherent optical properties and radiometric variables shows that use of phase functions with the correct backscatter fraction and overall shape is crucial to achieve model-data closure. (C) 2002 Optical Society of America
\end{abstract}

OCIS codes: $\quad 010.4450,290.1350$.

\section{Introduction}

The absorption coefficient $a$ and the volume scattering function (VSF) $\beta$ completely determine the inherent optical properties (IOPs) of a medium. Given $a$ and $\beta$ throughout a medium, along with suitable boundary conditions, the radiative transfer equation can be solved to obtain the radiance distribution within and leaving the medium. All other optical quantities of interest, such as irradiances and reflectances, then can be computed from the radiance. Oceanic absorption coefficients have been intensively studied and are routinely measured in situ as functions of depth and wavelength with commercially available instruments. However, the VSF is rarely measured in the ocean even though $\beta$ is fundamentally important to understand and predict oceanic radiance distributions and related quantities.

The VSF is a function of the (polar) scattering angle $\psi$, which is measured from 0 in the incident (unscattered or forward) direction. The integral of the VSF over all scattering directions gives the scattering coefficient $b$, which is a measure of the overall magnitude of scattering without regard to the angular

C. D. Mobley (mobley@sequoiasci.com) and L. K. Sundman are with Sequoia Scientific, Incorporated, 15317 NE 90th Street, Redmond, Washington 98052-3562. E. Boss is with Oregon State University, College of Ocean and Atmospheric Sciences, Corvallis, Oregon 97331.

Received 11 June 2001; revised manuscript received 18 October 2001.

0003-6935/02/061035-16\$15.00/0

(C) 2002 Optical Society of America pattern of the scattered light. The VSF is usually factored into $b$ times the scattering phase function $\tilde{\beta}=\mathrm{VSF} / b$, which specifies the angular dependence of the scattering without regard for its magnitude. The IOPs of the water body are then specified by $a, b$, and $\tilde{\beta}$.

The purpose of the present paper is to quantify the effects of the shape of the phase function $\tilde{\beta}$ at intermediate $(\psi \approx 5-90 \mathrm{deg})$ and backscatter $(\psi=$ 90-180 deg) angles on the radiometric quantities that are of broad interest in oceanography. Our approach uses various phase functions as input to the HYDROLIGHT 4.1 radiative transfer model ${ }^{1,2}$ (Sequoia Scientific, Inc.), which provides highly accurate numerical solutions of the unpolarized radiative transfer equation for the input phase function, other IOPs, and boundary conditions. For simplicity of this analysis, the water is taken to be homogeneous and infinitely deep. We finish with an example of the improvements that can be obtained in light-field predictions when phase functions having the correct backscatter fraction are employed.

In practice, the absorption coefficient $a$ and beam attenuation coefficient $c$ are usually measured, and the scattering coefficient is then obtained from $b=$ $c-a$. The relative contributions of absorption and scattering are conveniently expressed by the albedo of single scattering $\omega_{0}=b / c$, which gives the probability of scattering (rather than absorption) in any interaction of a photon with the medium. In ocean waters, $\omega_{0}$ can vary from 0.1 (absorption-dominated waters) to 0.9 (scattering-dominated waters) depending on wavelength and water composition. The unmeasured phase function $\tilde{\beta}$ is usually ap- 
proximated in one of three ways. First, any of several simple functional forms for $\tilde{\beta}$ can be used. Although these analytic approximations are mathematically convenient, they often give unrealistic phase functions, especially at small (near-forward) or large (near-backward) scattering angles. Second, Mie theory can be used to compute $\tilde{\beta}$ numerically. However, Mie theory requires as input the complex index of refraction (itself a function of wavelength) and size distribution of the scattering particles in the water body, and these quantities are themselves seldom measured. Moreover, Mie theory assumes that the particles are homogeneous and spherical, which is seldom the case for oceanic particles. Third, a phase function derived from one of the rare measurements of oceanic VSFs can be employed. The most commonly used VSF data set consists of eight VSFs measured by Petzold ${ }^{3}$ in 1971. An average $\tilde{\beta}$ derived from his data ${ }^{4}$ is used frequently in numerical radiative transfer studies. In any case, there is uncertainty about the adequacy of any assumed phase function to describe a particular water body under study. Given the dim prospects for routine measurement of the full VSF or of $\tilde{\beta}$ anytime in the near future, it is imperative to quantify the errors that may result in radiative transfer simulations of the marine environment from use of an incorrect phase function.

The remote-sensing reflectance $R_{\mathrm{rs}}$ is the ratio of the water-leaving radiance to the downwelling plane irradiance just above the sea surface. At the level of approximation expressed by singlescattering theory, ${ }^{5}$ scattered radiance and the remote-sensing reflectance depend on the phase function at a single scattering angle, which is determined by the directions of the incident and scattered beams. According to the quasi-singlescattering approximation, ${ }^{5}$ the remote-sensing reflectance depends only on the backscatter fraction $B=b_{b} / b$, which represents the probability that a photon will be scattered through an angle $\psi \geq 90$ deg in any scattering event. Here $b_{b}$ is the backscatter coefficient, which is the integral of the VSF over the hemisphere of backscattering directions $\psi \geq 90$ deg. However, when multiple scattering is taken into account, all radiometric quantities depend on the phase function over the full range of scattering angles. Different phase functions can have the same value for $B$, but the associated radiance distributions will differ because of differences in the actual shapes of $\tilde{\beta}(\psi)$. Thus there will be variability in radiometric quantities that is due to the $\psi$ dependence of the phase function. The magnitude of this variability will depend not just on the functional form of $\tilde{\beta}(\psi)$, but also on the importance of multiple scattering within the water (i.e., on $\omega_{0}$ ) and on external parameters such as the solar zenith angle. The same can be said of other quantities of interest.

Plass et al..$^{6}$ considered phase function effects on numerically computed radiance distributions and irradiances just above and below a level sea surface.
The two-term Henyey-Greenstein [TTHG; see Eq. (5) below] phase functions used in their simulations all had a backscatter fraction of $B=0.07$. They showed that changing the shape of the phase function can change upwelling radiances by factors of $2-10$, with $30 \%$ changes in the irradiance ratio $R=$ $E_{u} / E_{d}$. Although $B$ values of 0.07 can occur at blue wavelengths in clear ocean water, this value is larger by a factor of 3 or more than $B$ values for typical ocean waters, and the shape of the TTHG phase function does not agree closely with phase functions based on measurements or Mie calculations. Thus their results need to be reexamined with more realistic oceanic phase functions. Also, they did not consider phase function effects on the light field at depth.

Gordon ${ }^{7}$ considered the effects of uncertainty in the small-angle part of the phase function, which is the hardest part of $\tilde{\beta}$ to measure. He used a phase function based on Petzold's data from San Diego Harbor as input to Monte Carlo simulations of various irradiances and reflectances. Gordon found that knowledge of the phase function for scattering angles $\psi$ less than approximately $15 \mathrm{deg}$ is not necessary for prediction of plane irradiance diffuse attenuation $K_{d}$ or irradiance reflectance $R$, if errors of a few percent in these quantities are acceptable. He further concluded that measurement of $\beta(\psi)$ for $\psi \geq 15 \mathrm{deg}$ should be adequate for interpretation of upwelling irradiances $L_{u}$ as measured by the seaviewing wide field-of-view sensor (SeaWiFS) ocean color sensor. Gordon's simulations employed only one phase function, and he did not consider the effects of errors in $\tilde{\beta}(\psi)$ for scattering angles greater than 15 deg.

In Section 2 we define several phase functions, which are chosen for convenient comparison with a commonly used (Petzold) phase function. In Section 3 we use these phase functions to show that the exact shape of the phase function in backscatter directions is not critical to predictions of underwater light fields, so long as the phase function has the correct backscatter fraction and its overall shape is approximately correct. In Section 4 we show how measured backscatter fractions can be used to generate realistic analytical phase functions having the given backscatter fraction at each depth and wavelength, and in Section 5 we study the effects of backscatter fraction on underwater light fields. In Section 6 we illustrate the importance of using the correct phase function in numerical simulations by application of our modeling techniques to a comprehensive data set taken off the coast of New Jersey.

\section{Phase Functions}

Many different phase functions have been used in numerical simulations of marine light fields. A few of these are based on measured VSFs. Mie theory is often used to numerically generate phase functions corresponding to a given size distribution and optical properties of spherical particles. Analytical phase functions are frequently used because of their math- 

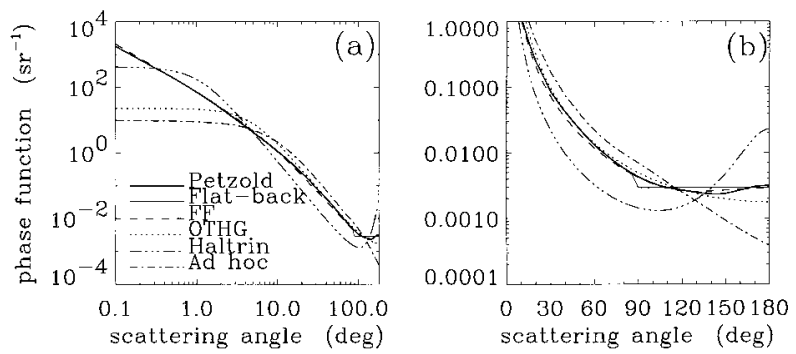

Fig. 1. Phase functions used in the HYDROLIGHT simulations of Section 2. Panel (a) is plotted to emphasize the small scattering angles; panel (b) is plotted to emphasize intermediate and large angles. Each phase function has a backscatter fraction of $B=$ 0.0183 . The curve patterns identify the phase functions as defined in Section 2.

ematical simplicity. We define here six phase functions for use in the HYDROLIGHT simulations. These particular phase functions are selected for their physical realism, mathematical properties, or historic precedence. Together these phase functions span the range of functional forms seen in the oceanographic literature.

\section{A. Petzold Average-Particle Phase Function}

This average-particle phase function is derived from three measurements of the VSF in San Diego Harbor; tabulated values are given in Mobley et al. ${ }^{4}$ and Mobley. ${ }^{8}$ When used in HYDROLIGHT, the tabulated values of $\log \tilde{\beta}$ versus $\log \psi$ are fit with a cubic spline to define the phase function at any scattering angle. When numerically integrated over $90 \leq \psi \leq 180 \mathrm{deg}$, this phase function gives a particle backscatter fraction of $B_{p}=0.0183$ (subscript $p$ on any symbol denotes the particle contribution to the total quantity). Because this phase function is based on observations and is so frequently used, we use it as the benchmark for defining and evaluating other phase functions. This phase function is plotted as the heavy solid curve in Fig. 1. Note that the Petzold phase function reaches a minimum near $\psi=140 \mathrm{deg}$ and then continues to increase with decreasing $\psi$ even at small scattering angles.

\section{B. Petzold Flat-Back Phase Function}

This phase function is almost the same as the Petzold average-particle phase function for $\psi \leq 90 \mathrm{deg}$. For $\psi \geq 90 \mathrm{deg}$, this phase function has a value of 0.0183 / $2 \pi=0.002913 \mathrm{sr}^{-1}$. Thus this phase function is flat (independent of $\psi$ ) for all backscatter directions, but it has the same $B_{p}$ as the Petzold average-particle phase function. This phase function is plotted in Fig. 1 as the light solid curve.

\section{Fournier-Forand Phase Function}

Fournier and Forand ${ }^{9}$ (FF) derived an approximate analytic form of the phase function of an ensemble of particles that have a hyperbolic (Junge-type) particle size distribution, with each particle scattering according to the anomalous diffraction approximation

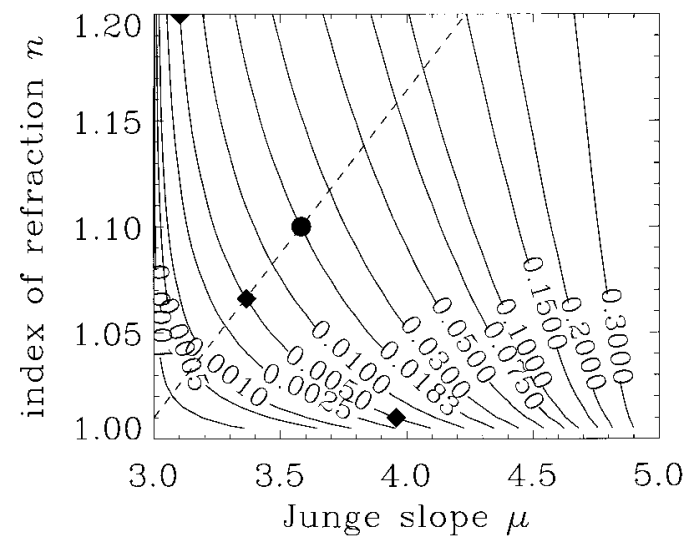

Fig. 2. Contours of the backscatter fraction $B_{p}$ of the FF phase function as determined by Eq. (3). The filled circle on the $B_{p}=$ 0.0183 curve shows the $(n, \mu)$ point that gives the best fit to the Petzold phase function of Fig. 1. The filled diamonds along the $B_{p}=0.005$ curve show the three $(n, \mu)$ pairs used to generate the inset in Fig. 7. The dashed line is Eq. (7).

to the exact Mie theory. In its latest form ${ }^{10}$ this phase function is given by

$$
\begin{aligned}
\tilde{\beta}_{\mathrm{FF}}(\psi)= & \frac{1}{4 \pi(1-\delta)^{2} \delta^{v}}\left[v(1-\delta)-\left(1-\delta^{v}\right)\right. \\
& \left.+\left[\delta\left(1-\delta^{v}\right)-v(1-\delta)\right] \sin ^{-2}\left(\frac{\psi}{2}\right)\right] \\
& +\frac{1-\delta_{180} v}{16 \pi\left(\delta_{180}-1\right) \delta_{180} v}\left(3 \cos ^{2} \psi-1\right),
\end{aligned}
$$

where

$$
v=\frac{3-\mu}{2}, \quad \delta=\frac{4}{3(n-1)^{2}} \sin ^{2}\left(\frac{\psi}{2}\right) .
$$

Here $n$ is the real index of refraction of the particles, $\mu$ is the slope parameter of the hyperbolic distribution, and $\delta_{180}$ is $\delta$ evaluated at $\psi=180 \mathrm{deg}$. Equation (1) can be integrated to obtain the particle backscatter fraction,

$$
B_{p}=1-\frac{1-\delta_{90}{ }^{v+1}-0.5\left(1-\delta_{90}{ }^{\nu}\right)}{\left(1-\delta_{90}\right) \delta_{90}{ }^{v}},
$$

where $\delta_{90}$ is $\delta$ evaluated at $\psi=90 \mathrm{deg}$. Although Eqs. (2) use only the real part of the index of refraction, the addition of moderate amounts of absorption does not significantly change the shape of the phase functions ${ }^{11}$ generated by Eq. (1).

Figure 2 shows selected backscatter values given by Eq. (3) as a function of $n$ and $\mu$. The filled circle on the $B_{p}=0.0183$ curve shows the values of $n=1.10$ and $\mu=3.5835$ for which Eq. (1) gives a good fit to the Petzold average-particle phase function. The corresponding $\tilde{\beta}$ plots in Fig. 1 (dashed curves) show that this FF phase function gives a good approximation to the Petzold phase function over the entire range of scattering angles. 


\section{One-Term Henyey-Greenstein Phase Function}

The one-term Henyey-Greenstein (OTHG) phase function is a one-parameter phase function ${ }^{12}$ that is frequently used because of its mathematical simplicity:

$$
\tilde{\beta}_{\mathrm{OTHG}}(\psi)=\frac{1}{4 \pi} \frac{1-g^{2}}{\left(1+g^{2}-2 g \cos \psi\right)^{3 / 2}} .
$$

The parameter $g$ is the mean cosine of the scattering angle. Equation (4) can be integrated over the backscatter directions to obtain

$$
B_{p}=\frac{1-g}{2 g}\left(\frac{1+g}{\sqrt{1+g^{2}}}-1\right) .
$$

A value of $g=0.9185$ gives $B_{p}=0.0183$. This phase function is plotted as a dotted curve in Fig. 1 . The OTHG phase function levels out for $\psi$ less than a few degrees, and it continues to decrease with increasing $\psi$ at larger angles; neither of these behaviors in seen in the measured Petzold phase function, or in the physically based FF phase function.

\section{E. Haltrin's Two-Term Henyey-Greenstein Phase Function}

Because the OTHG phase function gives a poor representation of oceanic phase functions at large and small scattering angles, a weighted sum of OTHG phase functions is sometimes used ${ }^{13}$ :

$$
\tilde{\beta}_{\mathrm{TTHG}}(\psi)=\alpha \tilde{\beta}_{\mathrm{OTHG}}\left(\psi, g_{1}\right)+(1-\alpha) \tilde{\beta}_{\mathrm{OTHG}}\left(\psi, g_{2}\right) \text {. }
$$

The parameter $g_{1}$ is given a value near 1 , which makes this TTHG phase function increase more strongly at small $\psi$ than does the OTHG phase function. The $g_{2}$ parameter is given a negative value, which makes the TTHG increase with angle as $\psi$ approaches $180 \mathrm{deg}$. The value of $\alpha, 0 \leq \alpha \leq 1$, gives the relative contributions of the two OTHG phase functions to the TTHG phase function. By considering integral relationships between the TTHG phase function and the mean cosine and cosine squared of the scattering angle, with regression to measured oceanic VSF data, Haltrin ${ }^{14}$ developed a version of the TTHG in which the $\alpha$ and $g_{2}$ parameters are given as functions of $g_{1}$ :

$$
\begin{aligned}
g_{2}= & -0.30614+1.0006 g_{1}-0.01826 g_{1}{ }^{2} \\
& +0.03644 g_{1}^{3} \\
\alpha= & \frac{g_{2}\left(1+g_{2}\right)}{\left(g_{1}+g_{2}\right)\left(1+g_{2}-g_{1}\right)} .
\end{aligned}
$$

Choosing $g_{1}=0.9809$ then gives $B_{p}=0.0183$ for the Haltrin TTHG phase function. The resulting phase function is plotted in Fig. 1. This phase function does behave somewhat better than the OTHG at small angles, but it is less by a factor of 3 or more than the Petzold phase function over the intermediate range of scattering angles from $\sim 10$ to $\sim 110 \mathrm{deg}$, and it is seven times the Petzold value at $\psi=180 \mathrm{deg}$.

\section{F. Ad Hoc Phase Function}

As just noted, Haltrin's TTHG has too little scattering at intermediate angles and too much at large angles when compared with the Petzold phase function. To aid in understanding the effects of these angle ranges, we defined an ad hoc phase function with the opposite behavior: higher scattering than Petzold at intermediate angles and much lower at large angles. This phase function was defined by trial and error; its tabulated values are fit by a cubic spline to define $\tilde{\beta}$ at other angles. The ad hoc phase function is plotted in Fig. 1. This phase function is somewhat unphysical when compared with measurements, especially at large and small scattering angles. However, its intended purpose is to bracket the spread of phase functions. We can anticipate that light fields computed with this phase function will show one extreme of behavior, and those computed with Haltrin's TTHG will show the other extreme, with the light fields computed with the other phase functions lying in between.

\section{G. Comparison of Phase Functions}

We can obtain a quantitative measure of the differences in these six phase functions by defining a rootmean-square (rms) percentage difference between two phase functions $\Delta_{\beta}$ as follows:

$$
\begin{aligned}
& \Delta_{\beta}= \\
& 100\left(\frac{1}{\psi_{2}-\psi_{1}} \int_{\psi_{1}}^{\psi_{2}}\left\{\frac{\tilde{\beta}_{1}(\psi)-\tilde{\beta}_{2}(\psi)}{\frac{1}{2}\left[\tilde{\beta}_{1}(\psi)+\tilde{\beta}_{2}(\psi)\right]} \sin \psi\right\}^{2} d \psi\right)^{0.5} .
\end{aligned}
$$

This definition normalizes the two phase functions by their average value at each scattering angle so as to prevent the rms difference from being dominated by the large magnitudes of the phase functions at small scattering angles. The sin $\psi$ factor weights each scattering angle by its importance in contributing to the scattering coefficients. Thus, for example, if two phase functions differ only near $\psi=180 \mathrm{deg}$, they will give nearly the same total and backscatter coefficients; in this sense they are similar. Likewise, the sine-weighted rms difference will also see these phase functions as being similar. If the $\sin \psi$ factor is omitted from the integrand, the rms difference gives a comparison of the two phase functions that is based only on the normalized shapes of the phase functions, with all scattering angles being viewed as equally important. Figure 1(a) shows that the six phase functions are coincidentally close together near $\psi=5 \mathrm{deg}$, which is therefore chosen as a convenient boundary between small and intermediate scattering angles. Table 1 shows both the sine-weighted and unweighted rms differences over intermediate angles $\psi=5-90$ deg, Table 2 over backscatter angles 90-180 deg, and Table 3 over 5-180 deg.

Because of its construction, flat back is closest to Petzold over the intermediate range of scattering angles. Of the remaining phase functions, either FF or 
Table 1. Rms Percentage Differences in Phase Functions, as Defined by Eq. (6), for Intermediate Scattering Angles $5 \leq \psi \leq 90 \mathrm{deg}^{a}$

\begin{tabular}{lrccccc}
\hline & \multicolumn{6}{c}{ Phase } \\
\cline { 2 - 7 } Function & Petzold & Flat Back & FF & OTHG & Haltrin & Ad Hoc \\
\hline Petzold & & 6.2 & 15.9 & 18.3 & 113.4 & 58.1 \\
Flat back & 6.2 & & 17.3 & 19.9 & 112.9 & 58.8 \\
FF & 9.3 & 11.2 & & 28.9 & 103.6 & 71.1 \\
OTHG & 7.1 & 11.0 & 14.4 & & 120.9 & 49.0 \\
Haltrin & 84.8 & 89.9 & 79.4 & 88.6 & & 146.0 \\
Ad hoc & 39.5 & 41.0 & 47.1 & 34.3 & 107.2 & \\
\hline
\end{tabular}

${ }^{a}$ The lower-left triangle of numbers is Eq. (6) including the sin $\psi$ factor in the integrand; the upper-right triangle of numbers is Eq. (6) without the $\sin \psi$ factor.

OTHG is the closest to Petzold, depending on whether the $\sin \psi$ factor is included in Eq. (6). By either version of Eq. (6), FF is closest to Petzold over backscatter angles. For the unweighted comparison, $\mathrm{FF}$ and flat back rank almost equal in closeness to Petzold when the full range of intermediate and backscatter angles is considered together. For the sine-weighted comparison, FF is closest, but not greatly different from flat back and OTHG. Ad hoc is closer to Petzold than is Haltrin for all angle ranges and both versions of Eq. (6). Haltrin and ad hoc of course always show the greatest rms differences of any pair of phase functions.

\section{Shape Effects}

We next consider the effect of the shape of the total phase function on the light field for a given backscatter fraction. In nature, the relative contributions of water and particles influence the shape of the total

Table 2. Rms Percentage Differences in Phase Functions, as in Table 1, for Backscattering Angles $90 \leq \psi \leq 180 \mathrm{deg}$

\begin{tabular}{lrrrrrr}
\hline & \multicolumn{6}{c}{ Phase Function } \\
\cline { 2 - 7 } Function & Petzold & Flat Back & FF & OTHG & Haltrin & Ad Hoc \\
\hline Petzold & & 15.5 & 6.4 & 26.1 & 88.8 & 83.6 \\
Flat back & 12.1 & & 11.7 & 34.7 & 85.3 & 90.4 \\
FF & 3.4 & 10.2 & & 27.6 & 87.9 & 85.1 \\
OTHG & 9.0 & 19.5 & 11.4 & & 102.3 & 66.2 \\
Haltrin & 50.6 & 45.9 & 49.1 & 56.4 & & 125.8 \\
Ad hoc & 31.4 & 40.7 & 33.7 & 24.1 & 68.2 & \\
\hline
\end{tabular}

Table 3. Rms Percentage Differences in Phase Functions, as in Table 1, for Intermediate and Backscattering Angles $5 \leq \psi \leq 180 \mathrm{deg}$

\begin{tabular}{lrrrrrr}
\hline & \multicolumn{5}{c}{ Phase Function } \\
\cline { 2 - 7 } Punction & Petzold & Flat Back & FF & OTHG & Haltrin & Ad Hoc \\
\hline Petzold & & 11.7 & 12.0 & 23.3 & 101.5 & 72.5 \\
Flat back & 9.4 & & 14.5 & 28.4 & 99.8 & 76.7 \\
FF & 7.0 & 10.4 & & 28.3 & 95.9 & 78.7 \\
OTHG & 8.1 & 15.6 & 12.9 & & 111.7 & 58.6 \\
Haltrin & 69.2 & 67.1 & 65.4 & 73.6 & & 136.0 \\
Ad hoc & 35.5 & 40.5 & 40.7 & 29.5 & 89.0 & \\
\hline
\end{tabular}

phase function, especially in backscatter directions, and give the total $B$ a wide range of values. The water contribution to the total backscatter can be quite significant at blue wavelengths in oligotrophic waters. Use of the particle phase functions defined above for the total phase function approximates a water body with a high particulate load, in which case the water contribution to the total phase function is small. In this section our intention is simply to compare phase function shape effects for one particular $B$ value, which is taken to be $B=0.0183$ for comparison with the commonly used Petzold phase function. Thus it is not necessary to consider here the relative contributions of water and particles to the total $\tilde{\beta}$. We simulate a particular water body in Section 6, where we account for the individual contributions of water, dissolved substances, and particles to the total IOPs.

The six phase functions just defined all have $B=$ 0.0183. According to Gordon's results, ${ }^{7}$ the large differences in these phase functions at scattering angles less than $\sim 5$ deg should affect downwelling plane irradiance calculations by at most a few percent. According to the quasi-single-scattering approximation, ${ }^{5}$ they would all give the same remotesensing reflectance; in reality they will give different values of $L_{u}$ or $R_{\mathrm{rs}}$. Multiple scattering will complicate the dependence of the light field on the phase function. We next study the extent to which the differences in these phase functions at intermediate and large scattering angles affect radiances and irradiances. When comparing results, we use the empirically based Petzold phase function as the benchmark for quantifying the results for the other five phase functions.

The six phase functions described above were used as input to HYDROLIGHT for a variety of IOP and environmental conditions. For simplicity, we modeled the water as homogeneous and infinitely deep. The IOPs were specified by the albedo of single scattering $\omega_{0}$ and phase function $\tilde{\beta}$; depth was measured as optical depth $\zeta=c z$. Simulations were performed for $\omega_{0}=0.2$ (highly absorbing, such as oligotrophic water at red wavelengths where absorption by the water itself dominates the particle contributions to the IOPs) and $\omega_{0}=0.9$ (highly scattering, such as at blue wavelengths in waters with a high load of both microbial and mineral particles). Solar zenith angles of $\theta_{s}=0$ (Sun overhead) and $60 \mathrm{deg}$ were used. We used HYDROLIGHT's default sky model, $, 2,15,16$ the sky radiance distribution that gives a directional pattern corresponding to a clear sky at a wavelength of 550 $\mathrm{nm}$; the corresponding spectral plane irradiance (Sun plus sky) onto the sea surface was $1.63 \mathrm{~W} \mathrm{~m}^{-2} \mathrm{~nm}^{-1}$ for $\theta_{s}=0$ and $0.71 \mathrm{~W} \mathrm{~m}^{-2} \mathrm{~nm}^{-1}$ for $\theta_{s}=60 \mathrm{deg}$. We modeled the sea surface using wave-slope statistics corresponding to a $5-\mathrm{m} \mathrm{s}^{-1}$ wind speed. These values cover the range of IOPs and environmental conditions commonly encountered in oceanographic studies.

Figure 3 shows the downwelling plane irradiances $E_{d}$ as a function of depth for the four combinations of 

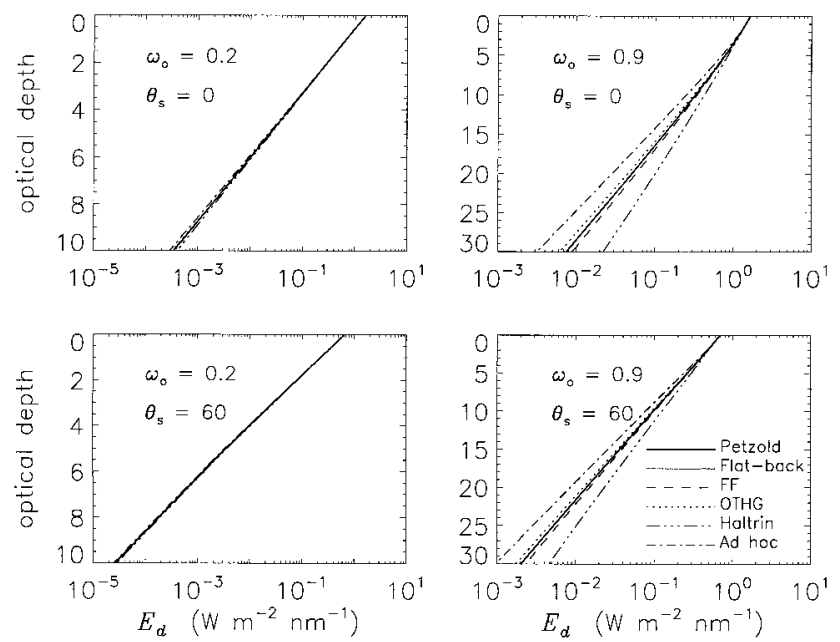

Fig. 3. Downwelling plane irradiances for four combinations of high or low scattering and high or low Sun angle. The line pattern identifies the phase function used in the calculations. The phase functions all have $B=0.0183$.

$\omega_{0}$ and $\theta_{s}$. For the high-absorption $\left(\omega_{0}=0.2\right)$ case, the bottom of the euphotic zone (defined here as the depth where the scalar irradiance, not shown, has decreased to 0.01 of its surface value) is at approximately $\zeta=5$ optical depths. The computed $E_{d}$ at $\zeta=$ 5 differ from the Petzold-computed values by only $\pm 1 \%$ for the three phase functions flat back, FF, and OTHG, which are the most similar to Petzold at medium and large scattering angles. The two extreme phase functions, Haltrin and $a d$ hoc, give irradiances at $\zeta=5$ that differ from Petzold by $\pm 6 \%$. For the high-scattering $\left(\omega_{0}=0.9\right)$ case, the bottom of the euphotic zone is at $\zeta=24$ to 27 for the Petzold phase function, depending on solar angle. At $\zeta=30$, the flat-back $E_{d}$ differs from Petzold by less than $1 \%$; FF and OTHG differ from Petzold by up to $19 \%$. The irradiances for the two extreme phase functions differ from Petzold by factors of $2-3$ at $\zeta=30$, depending on the phase function and solar angle. These results confirm the minimal importance of small-angle scattering, where OTHG differs from Petzold by an order of magnitude or more, and they highlight the importance of medium-angle scattering, where Haltrin and ad hoc differ considerably from Petzold. Note, for example, that for Haltrin the low scattering at medium angles scatters fewer photons from the incident beam into directions with large nadir angles than does Petzold and thus gives a greater value for $E_{d}$. For ad hoc, the greater scattering at medium angles scatters more photons from the incident beam into directions with large nadir angles and thus decreases $E_{d}$. These effects are more pronounced for the highscattering case.

Figure 4 shows the corresponding profiles for the upwelling radiance $L_{u}$. The Petzold, flat-back, and FF phase functions all show similar $L_{u}$ profiles, with flat-back and FF $L_{u}$ values at the surface usually being within $10 \%$ of the Petzold value. In the highabsorption, zenith-Sun case, the OTHG $L_{u}$ value is
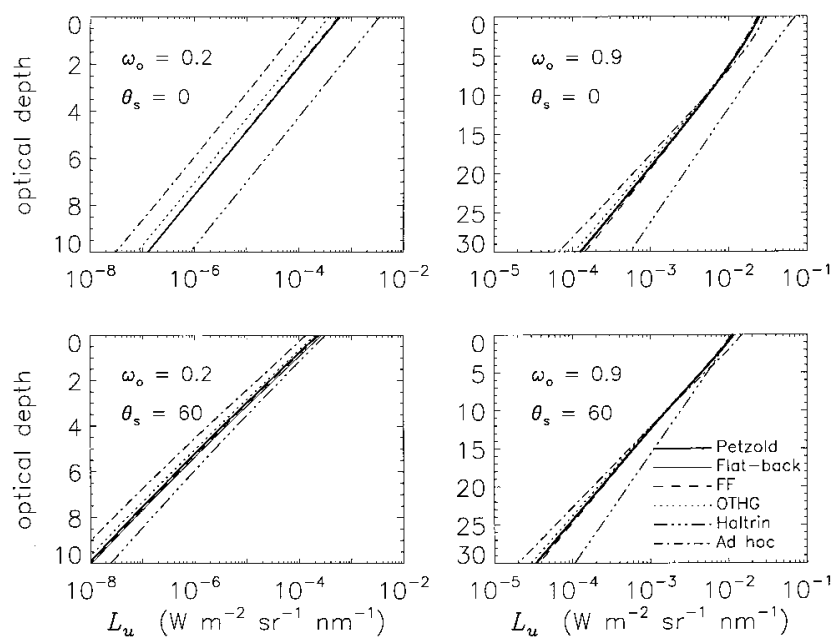

Fig. 4. Upwelling radiances corresponding to the simulations of Fig. 3.

$38 \%$ lower than the Petzold value, the Haltrin value is 5.7 times the Petzold value, and $a d$ hoc is too small by a factor of 4.3. In this high-absorption case, single scattering makes the primary contribution to $L_{u}$ near the surface; and for the Sun at the zenith, the relevant part of the phase function is the scattering angles near $180 \mathrm{deg}$. (In HYDROLIGHT, the $L_{u}$ radiances simulate a sensor with a top-hat response and a 5-deg half-angle field of view.) Thus the ratios of the $L_{u}$ values to the Petzold value are nearly proportional to the ratios of the respective phase functions near 180 deg, as can be seen in Fig. 1. A solar zenith angle of $60 \mathrm{deg}$ corresponds to $40 \mathrm{deg}$ in water, in which case the single-scattering contribution to $L_{u}$ is at a scattering angle of $\psi \approx 140 \mathrm{deg}$. The phase functions are closer together at this angle, so the $L_{u}$ values differ less than in the zenith-Sun case. Likewise, there is less difference in the high-scattering cases, for which multiple scattering makes a greater contribution to $L_{u}$, and thus the phase functions contribute to $L_{u}$ over a broad range of scattering angles, which tends to average out the differences at any one angle.

Figure 5 shows profiles of the irradiance reflectance $R=E_{u} / E_{d}$. This quantity involves light scattered into all directions and thus includes phase function effects from all scattering angles even in the high-absorption case. In the high-absorption, zenith-Sun case, flat back is 6\% greater than Petzold. This is a consequence of the somewhat greater backscattering by flat back between $\psi \approx 110$ and $165 \mathrm{deg}$; greater radiance scattered upward through these angles increases $E_{u}$ as compared with Petzold. This is the only simulation in which flat back and Petzold differ noticeably in their computed light fields. FF, which more closely matches Petzold for $\psi>90 \mathrm{deg}$, gives $R$ values within $1 \%$ of Petzold. In the highscattering cases, flat back is again almost indistinguishable in the plots from Petzold, and FF and OTHG are within 7\% of Petzold. The Haltrin and $\mathrm{ad}$ hoc phase functions give $R$ values that are, respec- 

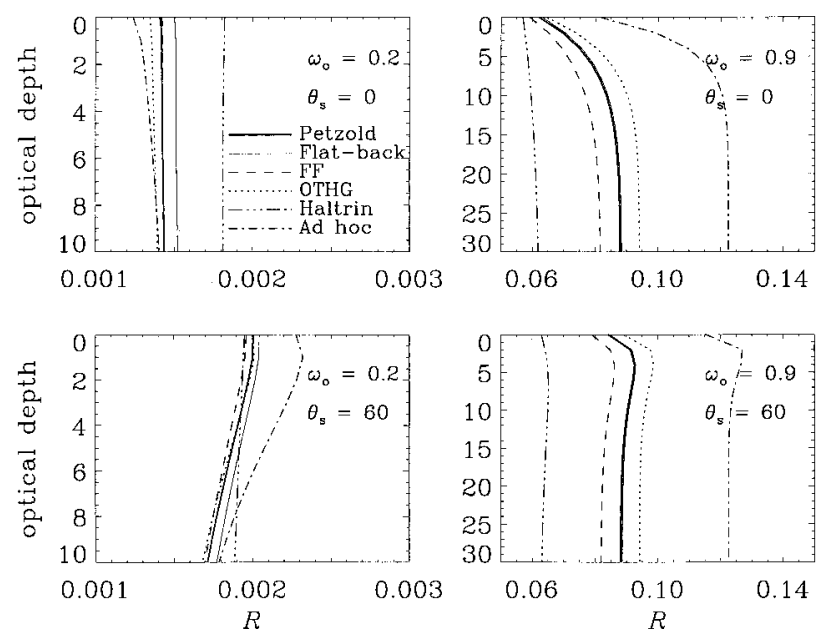

Fig. 5. Irradiance reflectances corresponding to the simulations of Fig. 3.

tively, up to $30 \%$ less and $39 \%$ greater than the Petzold values in the high-scattering cases. Note also that, for the high-absorption, zenith-Sun case, the Haltrin $R$ is higher than Petzold because of the peak in the Haltrin phase function near $180 \mathrm{deg}$, whereas the Haltrin values are lower than Petzold for larger Sun angles or higher scattering, which are affected by the lower scattering at intermediate scattering angles. The opposite behavior can be seen in the $a d$ hoc $R$ values.

Figure 6 shows profiles of the in-water remotesensing reflectance $R_{\mathrm{rs}}(z)=L_{u}(z) / E_{d}(z)$. According to single-scattering theory, this quantity is proportional to the phase function at a particular backscattering angle. For the high-absorption, zenith-Sun case, Petzold, flat back, and FF differ by less than 6\%; Haltrin and ad hoc show large deviations in direct proportion to their values at $\psi=180 \mathrm{deg}$. When the Sun is at $60 \mathrm{deg}$, the in-water scattering angle is near $140 \mathrm{deg}$, where there is less difference in the phase
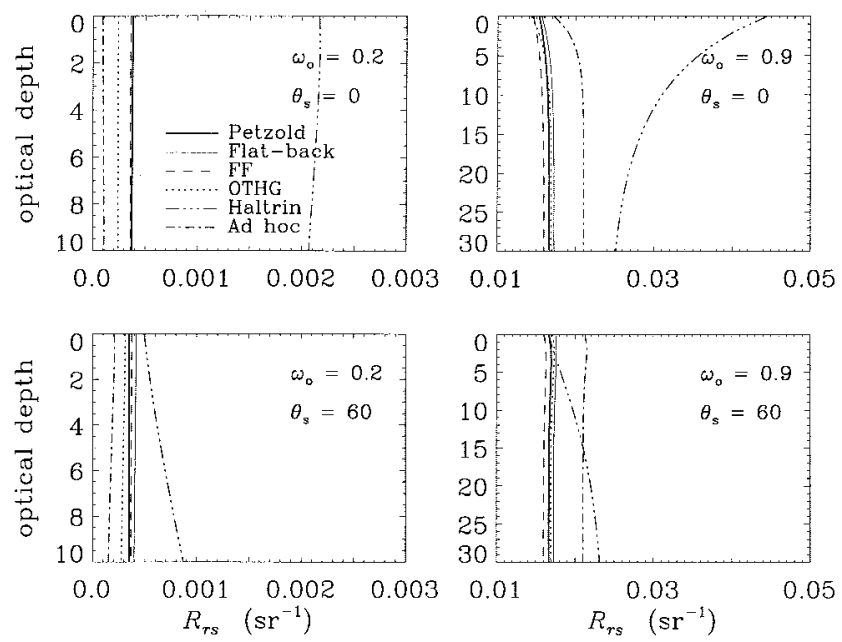

Fig. 6. Remote-sensing reflectances corresponding to the simulations of Fig. 3. functions and consequently less difference in $R_{\mathrm{rs}}$. In the high-scattering case, both Haltrin and ad hoc give greater $R_{\mathrm{rs}}$ values than the other phase functions, whose $R_{\mathrm{rs}}$ values vary among themselves by less than $9 \%$.

These simulations show that the exact shape of the phase function in the backscatter directions seldom makes more than a few percent difference in quantities such as $E_{d}, L_{u}, R$, and $R_{\mathrm{rs}}$, so long as the overall shape of the phase function does not differ greatly from the correct one (taken here to be Petzold). However, if the assumed phase function differs from the correct one by factors of 2 or more over intermediate and large scattering angles, as do the Haltrin and $a d$ hoc phase functions, then the errors in computed $E_{d}, L_{u}, R$, and $R_{\mathrm{rs}}$ values within the euphotic zone can be tens of percent or greater, depending on solar angle, depth, and the values of the absorption and scattering coefficients. The FF phase function generally gives quantities that agree with Petzold to within a few percent, which is better than other simple analytic phase functions such as OTHG and Haltrin's TTHG.

\section{Generating Phase Functions by Backscatter Value}

The preceding simulations show that the exact shape of the total phase function in the backscatter directions is not critical if errors of up to $10 \%$ are acceptable in computed light-field quantities. However, it is crucial that the phase function have the correct backscatter fraction $B$ when we compute the remotesensing reflectance or other quantities that are strongly influenced by backscattered radiance. In the past, numerical simulations often just assumedfor lack of actual measurements-that the Petzold phase function provides an adequate description of scattering by marine particles. Consequently the predicted light-field values correspond to a particle backscatter fraction of 0.0183 .

The ac-9 (WETLabs, Inc.; Ref. 17) is widely used to obtain in situ measurements of $a$ and $b=c-a$ as functions of depth $z$ and wavelength $\lambda$. Likewise, the HydroScat-6 (HOBI Labs, Inc.; Ref. 18) and ECOVSF (WETLabs, Inc.; Ref. 19) instruments can be used to estimate $b_{b}(z, \lambda)$ from in situ measurements of the VSF at selected angles in the backscatter directions. It is thus possible to make hydrographic casts in which (after removal of the water contributions) $B_{p}(z, \lambda)=b_{b p}(z, \lambda) / b_{p}(z, \lambda)$ is obtained simultaneously with measurements of the other IOPs and of the light field. Incorporating such $B_{p}(z, \lambda)$ information into radiative transfer simulations can be expected to give increased accuracy in the predictions of marine light fields. However, because $B_{p}$ generally varies with depth and wavelength, so must the particle phase function used in numerical models. We therefore next develop a numerically efficient way to generate a new phase function at each depth and wavelength as the numerical model solves the radiative transfer equation. This is referred to as dynamic generation of the phase function, as opposed to 
the a priori selection of the phase function before the computations begin.

The FF phase function is physically grounded in Mie theory and reasonable assumptions about the index of refraction and particle size distribution of oceanic particles. As can be seen in Fig. 1, it consequently gives better approximations to measured oceanic particle phase functions than does any other commonly used analytical phase function. The resulting light-field quantities are in agreement to $\sim 10 \%$ or better with those computed with the empirical Petzold phase function for the case of $B=0.0183$. Moreover, analytical formulas for other quantities of interest, such as the backscatter fraction and the cumulative probability distribution function for scattering (which is needed for Monte Carlo simulations), can be obtained for the FF phase function. ${ }^{10}$ This phase function is therefore ideal to generate phase functions having any needed value for the backscatter fraction $B_{p}$.

According to Eqs. (2) and (3), there is no unique pair of $n$ and $\mu$ values corresponding to a given $B_{p}$. We therefore must decide which $n$ and $\mu$ values to use in Eqs. (2) to generate the $v$ and $\delta$ inputs to Eq. (1). The needed $n$ and $\mu$ can be determined by ancillary analysis of particle properties and measurements of the particle size distribution, but such data are rarely available. Twardowski et al. ${ }^{20}$ have developed a method of estimating $n$ and $\mu$ from measurements of the spectral particle beam attenuation coefficient $c_{p}(\lambda)$ and particle backscatter fraction $B_{p}$. Their technique is convenient because it relies only on IOP data obtainable from instruments such as the ac-9 and HydroScat-6. These $n$ and $\mu$ values can be used to generate the corresponding FF phase function. However, the method of Twardowski et al. ${ }^{20}$ cannot be used if $c_{p}$ is measured at only one wavelength. We therefore seek another way to determine $n$ and $\mu$ values corresponding to a given $B_{p}$ value.

It is reasonable to assume that oceanic particles having $B_{p}$ values less than $\sim 0.01$ are living microbes with indices of refraction between 1.01 and 1.09 (relative to water), whereas particles having $B_{p}$ values greater than a few percent are mineral particles with indices of refraction of the order of $1.15 .^{21-23}$ The best-fit value of $n=1.10$ for the Petzold phase function used here is consistent with this idea if the San Diego Harbor waters were a mixture of microbes and resuspended sediments. For simplicity we assume a linear relationship between $n$ and $\mu$ that reproduces the FF fit to the Petzold data (the filled circle shown in Fig. 2) and that uses $n=1.01$ at the smallest value of $\mu$ (i.e., at the smallest value of $B_{p}$ ). This function, which is shown by the dashed line in Fig. 2, is given by

$$
n=1.01+0.1542(\mu-3),
$$

or equivalently,

$$
\frac{2}{3 \delta_{90}}=(0.01-0.3084 v)^{2} .
$$

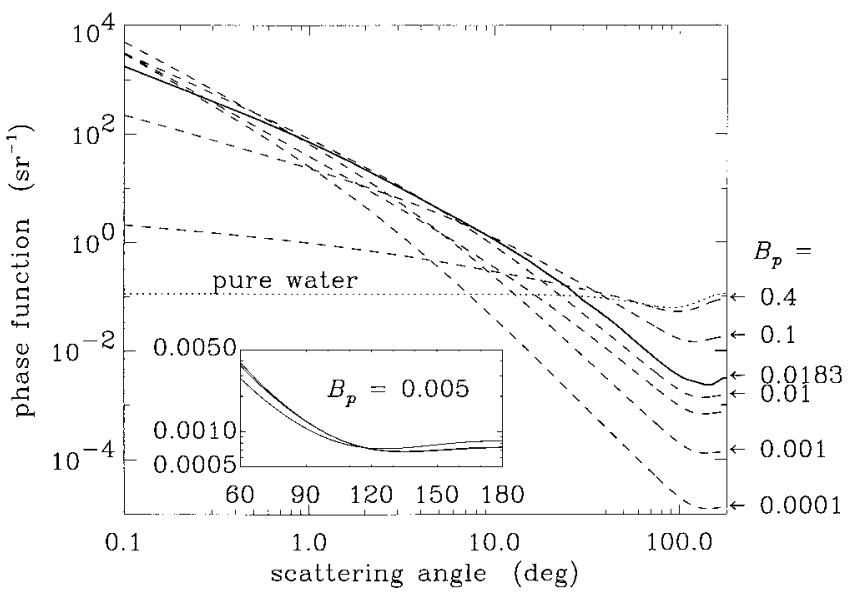

Fig. 7. FF phase functions as generated by Eq. (1) for different backscatter fractions $B_{p}$ (dashed curves). The corresponding ( $n$, $\mu$ ) values lie along the dashed line of Fig. 2 . The solid curve with $B_{p}=0.0183$ is the Petzold average-particle phase function. The pure-water phase function (dotted curve) has $B=0.5$. The inset shows the three phase functions corresponding to the three sets of $(n, \mu)$ values shown by filled diamonds on the $B_{p}=0.005$ contour of Fig. 2.

Given a value of $B_{p}$, Eqs. (3) and (8) can be solved numerically to obtain $\delta_{90}$ and $\nu$, from which $n$ and $\mu$ are obtained from Eqs. (2). Figure 7 shows the family of $\mathrm{FF}$ phase functions generated in this manner for a wide range of $B_{p}$ values.

As can be seen in Fig. 2, Eq. (7) yields a particular pair of $n$ and $\mu$ values for a given $B_{p}$. However, any other $(n, \mu)$ pair lying along the line of constant $B_{p}$ as can be seen in Fig. 2 also would be mathematically acceptable. The phase functions for a given $B_{p}$ but different $n$ and $\mu$ values will have somewhat different shapes. We therefore must consider whether the differences in the FF phase functions generated by different $n$ and $\mu$ values for a given $B_{p}$ are important. The inset in Fig. 7 shows the three FF phase functions generated for the three pairs of $(n, \mu)$ values shown by the filled diamond symbols on the $B_{p}=$ 0.005 line of Fig. 2. Visually, there is nothing to recommend one over the others. When compared with the sine-weighted rms difference of Eq. (6), these phase functions differ by less than $20 \%$ over the $5-180$-deg range and by less than $7 \%$ over the $90-$ 180-deg range. Similar results hold for other $B_{p}$ values. Thus the choice of $n$ and $\mu$ values for a particular $B_{p}$ does not appear to be critical, and the values lying along the dashed line of Fig. 2 are satisfactory.

The FF phase functions in Fig. 7 and additional phase functions for intermediate $B_{p}$ values are available in a precomputed format in HYDROLIGHT 4.1. As HYDROLIGHT runs using, for example, input files of ac-9 and HydroScat- 6 data to determine $B_{p}(z, \lambda)$ at each depth and wavelength, this library of FF phase functions is used dynamically to look up a phase function with the needed $B_{p}(z, \lambda)$ value. For $B_{p}$ values not equal to the values found in the library, linear inter- 

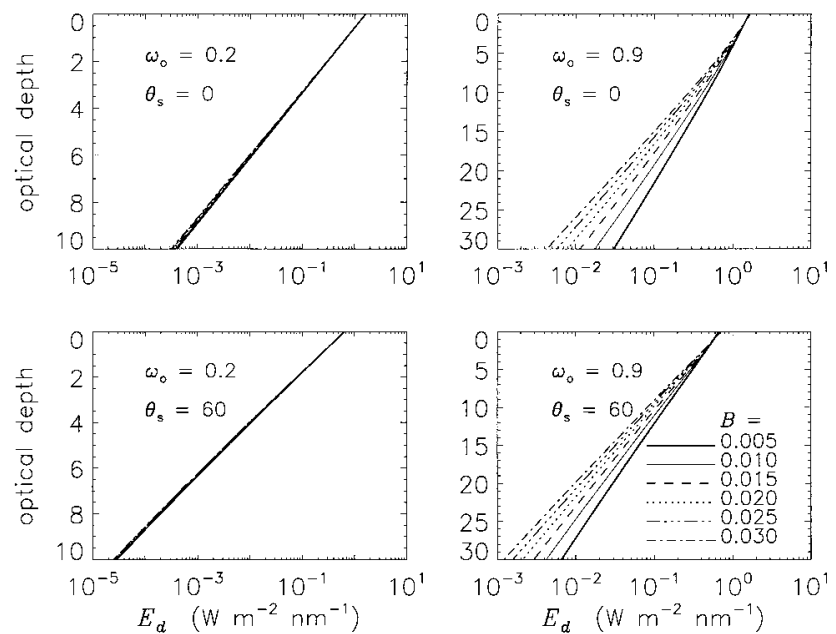

Fig. 8. Downwelling plane irradiances for four combinations of high or low scattering and high or low Sun angle. The line pattern identifies the backscatter fraction $B$ of the FF phase function used in the calculations.

polation is performed with the tabulated phase functions with $B_{p}$ values just above and below the needed value. This proves to be computationally efficient, and the look-up table is much smaller than one based on the full range of possible $n$ and $\mu$ values.

\section{Backscatter Effects}

Gordon ${ }^{7}$ showed that, for a given phase function, truncation of the phase function at small scattering angles does not greatly affect $E_{d}$ or $R$. However, this does not imply that $E_{d}$ and other quantities are nearly independent of the phase function. The simulations of Section 3 are consistent with Gordon's conclusion about the unimportance of small scattering angles, but they showed that the shape of the phase function at intermediate scattering angles can have a large influence on the light field.

Figure 8 shows $E_{d}$ as computed with $\mathrm{FF}$ phase functions having backscatter fractions from 0.005 to 0.03 ; such $B$ values are typical of oceanic waters. As in Section 3, we use the FF particle phase function as the total. Changing the $B$ value of course changes the shape of the FF phase function at all angles, as can be seen in Fig. 7. Figure 8 shows that, for absorption-dominated waters, the phase function has minimal effect on $E_{d}$. However, when scattering dominates, $E_{d}$ depends on $B$, i.e., on the shape of the $\mathrm{FF}$ phase function.

Figure 9 shows the corresponding upwelling radiances. As expected, $L_{u}$ near the surface is directly proportional to $B$. This factor-of-six difference in $L_{u}$ changes little with depth in the high-absorption case, for which single scattering makes the primary contribution to $L_{u}$. For the high-scattering case, $L_{u}$ decays with depth at noticeably different rates for different $B$. Figure 10 shows the corresponding remote-sensing reflectances. $R_{\mathrm{rs}}$ is directly proportional to $B$ and there is little variation induced by boundary effects near the sea surface. The corre-
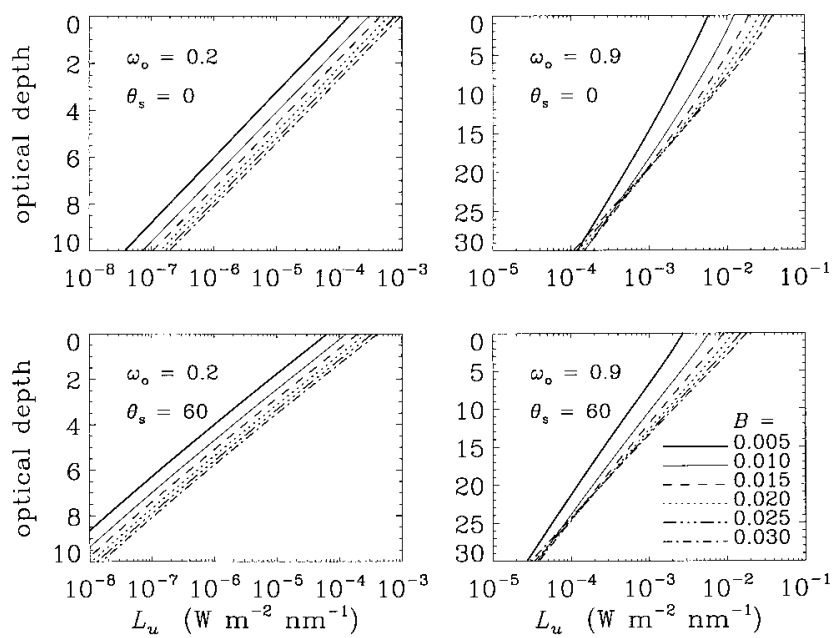

Fig. 9. Upwelling radiances corresponding to the simulations of Fig. 8.

sponding curves for $R$ (not shown) are similar to those for $R_{\mathrm{rs}}$, although there are noticeable boundary effects in the $R$ values for the first few optical depths. In any case, we again see the importance of having a phase function with the correct backscatter fraction.

\section{Application}

The preceding simulations show that, although the small-angle dependence of the phase function is not important in computations of $E_{d}$, use of a phase function with the correct angular dependence at intermediate scattering angles is crucially important even in predictions of $E_{d}$. Likewise, the phase function must have the correct backscatter fraction, although the detailed shape of the phase function at backscatter angles is less important. We next illustrate the effect of use of dynamically generated phase functions having the proper $B_{p}$ value, as opposed to use of a Petzold phase function at all depths and wavelengths as is often done.
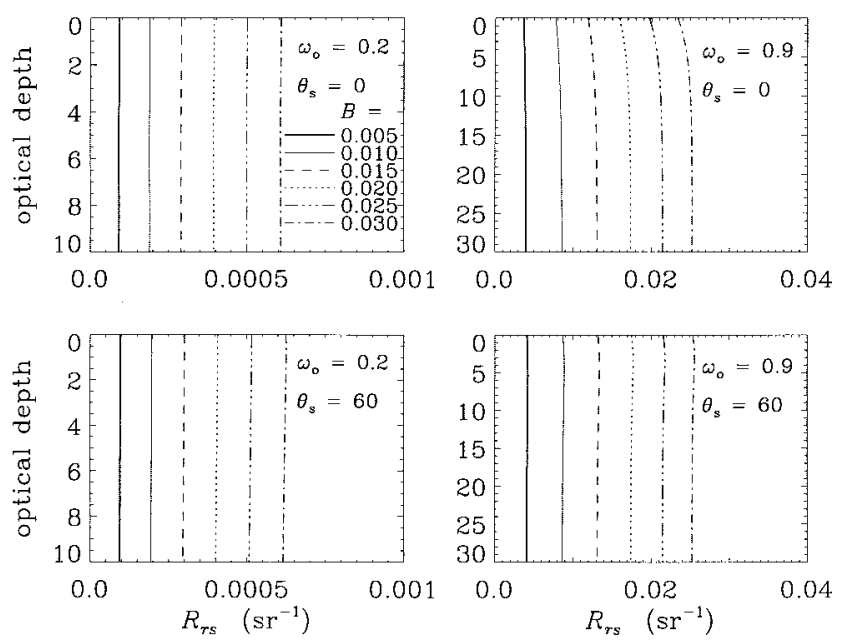

Fig. 10. In-water remote-sensing reflectances corresponding to the simulations of Fig. 8. 
Table 4. Data Taken at the LEO-15 Site as Used to Model the In-Water Light Field ${ }^{a}$

\begin{tabular}{lll}
\hline \multicolumn{1}{c}{ Quantity Measured } & \multicolumn{1}{c}{ Instrument } & Nominal Wavelength $(\mathrm{nm})$ \\
\hline Total $a(z, \lambda)$, total $c(z, \lambda)$ & Unfiltered ac-9 & $412,440,488,532,555,650,676,715$ \\
Dissolved $a(z, \lambda)$ & Filtered ac-9 & $412,440,488,532,555,650,676,715$ \\
Backscatter $b(z, \lambda)$ derived from & HydroScat-6 & $442,488,532,555,620$ \\
$\quad$ VSF at $\psi=140$ deg & ECO-VSF & \\
Backscatter $b(z, \lambda)$ derived from & & 530 \\
$\quad$ VSF at $\psi=100,125$, and 150 deg & VSM & 530 \\
VSF $(\psi=0.6-179.6$ deg) & OCP & $412,443,489,533,555,591,683$ \\
$E_{d}(z, \lambda)$ and $L_{u}(z, \lambda)$ & Multichannel visible & $412,443,489,533,555,591,683$ \\
Sky $E_{d}(\lambda)$ & detector system & 123 wavelengths between 396 and 798 \\
Sky $E_{d}(\lambda)$ and $L_{u}(z=0.6 \mathrm{~m}, \lambda)$ & Hyper-TSRB &
\end{tabular}

${ }^{a}$ Most instruments have a nominal $10-\mathrm{nm}$ bandwidth centered on the listed wavelengths.

A comprehensive data set of IOPs, radiometric variables, and ancillary oceanographic data was collected during the summer 2000 field campaign of the Hyperspectral Coastal Ocean Dynamics Experiment at the LEO-15 site off the coast of New Jersey. For our modeling purposes here, we select data taken from nearly simultaneous hydrographic casts made near local noon on 24 July 2000 at $39^{\circ}$ 24.91' North, $74^{\circ} 11.78^{\prime}$ West (Station 19). One cast measured absorption and beam attenuation coefficients with filtered and unfiltered ac-9's and backscatter coefficients with HydroScat-6 and ECO-VSF instruments on a slow-drop package. The VSF was measured simultaneously at a $2-\mathrm{m}$ depth at an approximately $10-\mathrm{m}$ horizontal distance from the profiling instruments by the recently developed volume scattering meter (VSM) instrument. ${ }^{24}$ A second cast measured $E_{d}$ and $L_{u}$ depth profiles with an ocean color profiler (OCP; Satlantic, Inc.). This package also contained an ac-9 for comparison with the one on the slow-drop package. Above-surface spectral $E_{d}$ and below-surface spectral $L_{u}$ were measured by a hyperspectral tethered spectral radiometer buoy (Hyper-TSRB; Satlantic, Inc.). The incident sky irradiance was also monitored with a multichannel visible detector system; (Satlantic, Inc.) on the nearby ship. Table 4 lists the optical data taken. The sky was overcast and the wind speed was estimated at $6 \mathrm{~m} \mathrm{~s}^{-1}$.

The ac-9 data were measured as described in Twardowski et al. ${ }^{25}$ The scattering correction applied to the ac-9 absorption coefficient was the third method of Zaneveld et al., ${ }^{26}$ in which a portion of $b$ was subtracted from $a$, with the assumption that $a=0$ at $715 \mathrm{~nm}$ (after subtraction of pure-water values). The HydroScat and ECO-VSF data were acquired and processed in accordance with the manufacturers' instructions, including correction for attenuation. In the simulations below, we used the data from the ac-9's that were on the slow-drop package because those data were obtained simultaneously with the backscatter data.

Figure 11 shows the measured IOPs as a function of depth and wavelength. In all cases, pure-water values were subtracted out. Approximately $40 \%$ of the particulate plus dissolved absorption at $412 \mathrm{~nm}$ $\left[a_{p+\mathrm{CDOM}}\right.$, Fig. 11(a), as measured by the unfiltered ac-9] is caused by colored dissolved organic matter $(\mathrm{CDOM})\left[a_{\mathrm{CDOM}}\right.$, Fig. $11(\mathrm{~b})$, as measured by the filtered ac-9] and $60 \%$ is caused by particles $\left[a_{p}\right.$, Fig. 11 (c)]. Figure 11(d) shows the particulate scattering coefficient, and Fig. 11(e) shows the particulate plus dissolved beam attenuation. Figure 11(e) also shows the chlorophyll concentration, which was estimated from the particulate absorption by ${ }^{27}$

$$
\begin{aligned}
\mathrm{Chl}= & {\left[a_{p}(676 \mathrm{~nm})-a_{p}(650 \mathrm{~nm})\right] } \\
& / 0.014\left(\mathrm{mg} \mathrm{m}^{-3}\right) .
\end{aligned}
$$

In the upper few meters of the water column, $\mathrm{Chl} \approx$ $6.7 \mathrm{mg} \mathrm{m}^{-3}$. According to commonly used biooptical IOP models for case I waters, ${ }^{28,29}$ a Chl value of $6.7 \mathrm{mg} \mathrm{m}^{-3}$ would give $a_{\mathrm{CDOM}} \approx 0.06 \mathrm{~m}^{-1}$ and
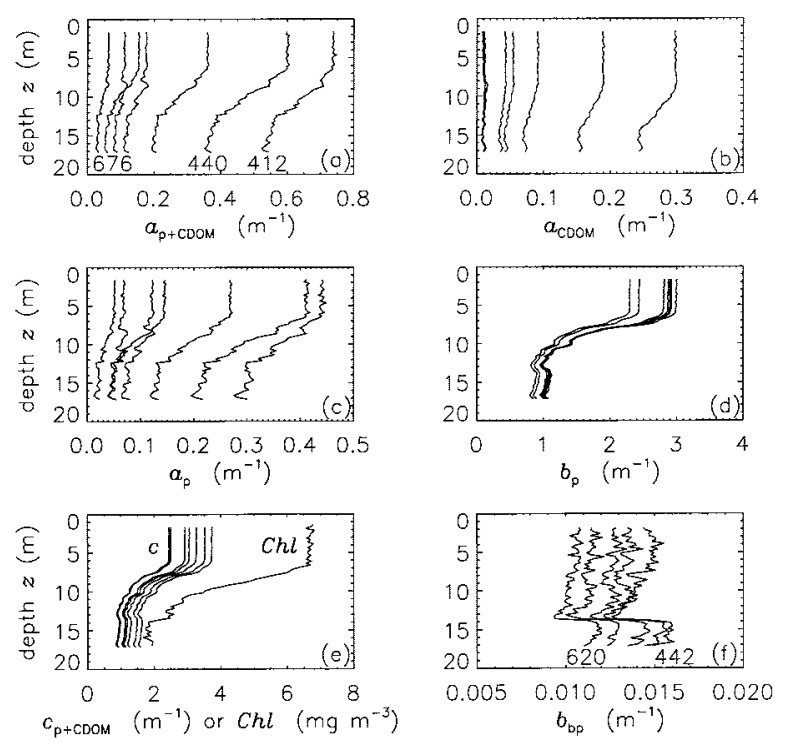

Fig. 11. Measured IOPs as a function of depth and wavelength, as labeled on the abscissa. (a)-(e) The seven wavelengths are 412 , $440,488,532,555,650$, and $676 \mathrm{~nm}$, with wavelength generally increasing from right to left in the plot, as indicated in (a). (f) The wavelengths are 442, 488, 532, 555, and $620 \mathrm{~nm}$, from right to left. 


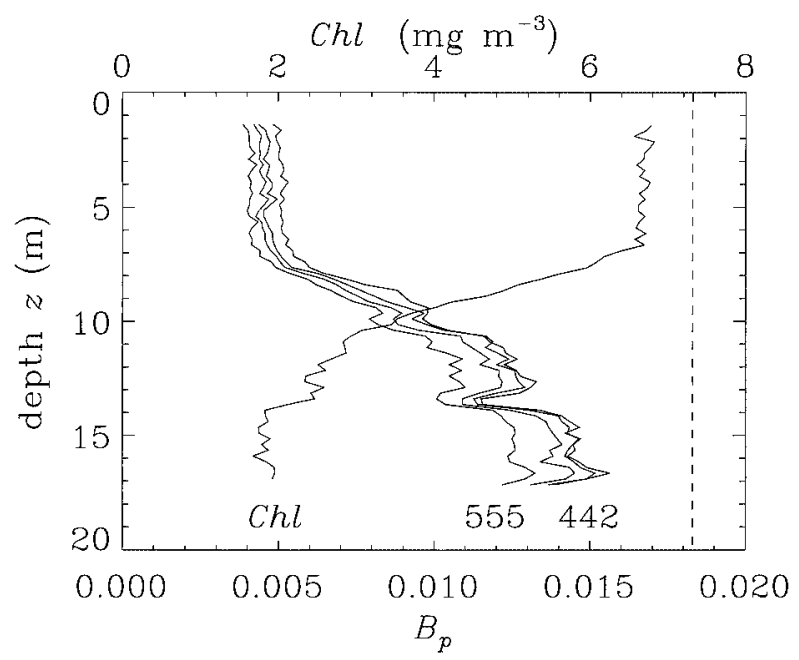

Fig. 12. Particulate backscatter fraction $B_{p}$ determined from Figs. 11(d) and 11(f). The wavelengths shown are 442, 488, 532, and $555 \mathrm{~nm}$, from right to left. The chlorophyll concentration is also shown to highlight the correlation between $B_{p}$ and Chl. The dashed line at 0.0183 indicates the backscatter fraction of the Petzold phase function.

$a_{p} \approx 0.18 \mathrm{~m}^{-1}$ at $412 \mathrm{~nm}$. Likewise, the particulate scattering coefficient $b_{p}$ that can be seen in Fig. $11(\mathrm{~d})$ is over twice the value expected $\left(1.3 \mathrm{~m}^{-1}\right)$ at $412 \mathrm{~nm}$ for case I water. Thus this water can be characterized as biologically productive, case II water having a relatively high CDOM concentration derived from terrigenous sources (the Hudson River and Great Bay, New Jersey). Finally, Fig. 11(f) shows the particulate backscatter coefficient (measured with the HydroScat-6). The $b_{b p}$ data at 676 $\mathrm{nm}$ are omitted because of possible contamination by self-induced chlorophyll fluorescence. ${ }^{30}$ Unlike the total scattering, the backscatter profiles show little correlation with the chlorophyll profile. This is likely due to the fact that total scattering $b_{p}$ and backscatter $b_{b p}$ are influenced by particle concentration and composition in different ways. Thus $b_{p}$ correlates strongly with the chlorophyll concentration (i.e., with particle concentration), whereas the presence of resuspended, high-index-of-refraction sediments below $14 \mathrm{~m}$ is likely responsible for the jump in $b_{b p}$ at $14 \mathrm{~m}$, which is much less noticeable in $b_{p}$.

Dividing the particulate backscattering coefficients of Fig. 11(f) by the particulate scattering coefficients of Fig. 11(d) gives the particulate backscatter fraction $B_{p}(z, \lambda)$; the result is shown in Fig. 12 for the four common ac-9 and HydroScat-6 wavelengths of 442, 488,532 , and $555 \mathrm{~nm}$. In the high-chlorophyll surface water the backscatter fraction is 0.004-0.005, depending on wavelength. As Chl decreases with depth to approximately $2 \mathrm{mg} \mathrm{m}^{-3}$ at $14 \mathrm{~m}, B_{p}$ increases in magnitude because of the decrease in the particulate total scattering coefficient. This increase in $B_{p}$ with depth is consistent with the reasonable supposition that near the surface the
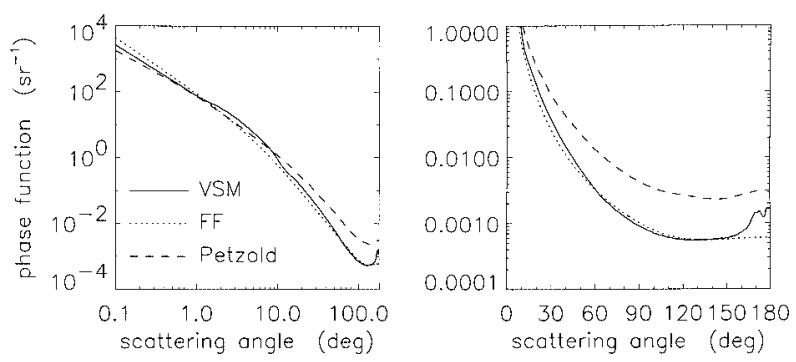

Fig. 13. Phase functions used in HYDROLIGHT simulations of the LEO-15 data. Solid curve, phase function measured by the VSM at $z=2 \mathrm{~m}, \lambda=530 \mathrm{~nm}$; dotted curve, FF phase function with the same backscatter fraction (0.00415); dashed curve, Petzold phase function with $B=0.0183$.

particles are predominately phytoplankton, which have low backscatter; but that at depth there is an increasing fraction of mineral particles from resuspended sediments, which have a high backscatter fraction. The percent decrease in $B_{p}$ from 442 to 555 $\mathrm{nm}$ is less than $24 \%$ at all depths.

The VSF at $530 \mathrm{~nm}$ and 2-m depth was measured from $\psi=0.6$ to $177.3 \mathrm{deg}$ by $0.3 \mathrm{deg}$ with the VSM instrument. To compute the total scattering coefficient, the measured VSF was extrapolated to $\psi=0$ by the model

$$
\operatorname{VSF}(\psi)=\operatorname{VSF}\left(\psi_{0}\right)\left(\frac{\psi_{0}}{\psi}\right)^{S}
$$

which is a linear extrapolation on a log VSF-log $\psi$ plot. The slope $S=1.538$ was determined from the measured VSF values at $\psi=0.6$ and $1.2 \mathrm{deg}$. We extended the VSF to 180 deg by setting $\operatorname{VSF}(180)=$ $\mathrm{VSF}(177.3)$. Integrating Eq. (9) analytically from $\psi=0$ to $0.6 \mathrm{deg}$ and then integrating $\operatorname{VSF}(\psi) \mathrm{nu}-$ merically from 0.6 to 180 deg gives a scattering coefficient of $b_{p}=2.40 \mathrm{~m}^{-1}$. Integrating from 90 to $180 \mathrm{deg}$ gives $b_{b p}=0.010 \mathrm{~m}^{-1}$. These $b_{b p}$ and $b_{p}$ values give a backscatter fraction of 0.00415 , which is in excellent agreement with the value of 0.00420 obtained from the HydroScat and ac-9 instruments, as can be seen in Fig. 12 for this depth and wavelength. Using the ECO-VSF backscatter measurement at 530 (not shown) in place of the HydroScat measurement, we obtain a value of $B_{p}=0.0047$. Thus three independent determinations of $B_{p}(z=2$ $\mathrm{m}, \lambda=530 \mathrm{~nm}$ ) are in agreement to within $8 \%$ of the average.

Figure 13 shows the phase function determined from the measured VSF, the FF phase function having the same $B_{p}$ value, 0.00415 , and the Petzold phase function with $B_{p}=0.0183$. At scattering angles from 10 to $52 \mathrm{deg}$, the FF phase function is as much as $35 \%$ less than the measured phase function; between 52 and $157 \mathrm{deg}, \mathrm{FF}$ is within $\pm 12 \%$ of the measured values; and FF is $64 \%$ less than measured at $177.3 \mathrm{deg}$. Tables $5-7$ give the quantitative rms comparisons of these three phase functions with the metrics of Eq. (6). On the log plot of Fig. 13 , the measured and FF phase functions are visu- 
Table 5. Rms Percentage Differences in the Three Phase Functions of Fig. 13, as Defined by Eq. (6), for Intermediate Scattering Angles $5 \leq \psi \leq 90 \operatorname{deg}^{a}$

\begin{tabular}{cccr}
\hline & \multicolumn{3}{c}{ Phase Function } \\
\cline { 2 - 4 } Phase Function & Petzold & VSM & FF \\
\hline Petzold & & 103.7 & 113.0 \\
VSM & 86.5 & & 26.9 \\
FF & 87.7 & 12.4 & \\
\hline
\end{tabular}

${ }^{a} \mathrm{VSF}$ is the measured phase function, and $\mathrm{FF}$ is the FournierForand phase function with the same backscatter fraction. The lower-left triangle of numbers is Eq. (6) including the sin $\psi$ factor in the integrand; the upper-right triangle of numbers is Eq. (6) without the $\sin \psi$ factor.

ally close except near $180 \mathrm{deg}$, and both are quite distinct from the Petzold phase function.

We used the IOPs of Fig. 11 as input to HYDROLIGHT. The total IOPs were built up as the sum of contributions by pure water, CDOM, and particles. Purewater absorption coefficients were obtained from Pope and Fry ${ }^{31}$ and scattering coefficients from Morel. ${ }^{32}$ CDOM was assumed to be nonscattering. Therefore partitioning the absorption into particulate and dissolved components as seen in Fig. 11 had no impact on modeling the elastic-scattering part of the radiance distribution. However, knowing the absorption by CDOM was necessary to include fluorescence by CDOM. Likewise, knowing the chlorophyll profile was necessary for the inclusion of chlorophyll fluorescence, even though Chl was not used to determine the IOPs. Raman scatter by water itself was also included in the simulations. The incident sky spectral irradiance was taken from the Hyper-TSRB values, and we modeled the sky radiance distribution as a solid overcast using the default sky radiance models available in HYDROLIGHT. We modeled the sea surface using wave-slope statistics for a $6-\mathrm{m} \mathrm{s}^{-1}$ wind. HYDROLIGHT was run with 27 wavelength bands whose

Table 6. Rms Percentage Differences in the Three Phase Functions of Fig. 13, as in Table 5, for Backscattering Angles $90 \leq \psi \leq 180 \mathrm{deg}$

\begin{tabular}{cccr}
\hline & \multicolumn{3}{c}{ Phase Function } \\
\cline { 2 - 4 } Phase Function & Petzold & VSM & FF \\
\hline Petzold & & 118.4 & 127.4 \\
VSM & 90.1 & & 34.4 \\
FF & 88.3 & 7.0 & \\
\hline
\end{tabular}

Table 7. Rms Percentage Differences in the Three Phase Functions of Fig. 13, as in Table 5, for Intermediate and Backscattering Angles $5 \leq \psi \leq 180 \mathrm{deg}$

\begin{tabular}{cccr}
\hline & \multicolumn{3}{c}{ Phase Function } \\
\cline { 2 - 4 } Phase Function & Petzold & VSM & FF \\
\hline Petzold & & 111.4 & 120.4 \\
VSM & 88.1 & & 31.0 \\
FF & 87.8 & 10.0 & \\
\hline
\end{tabular}
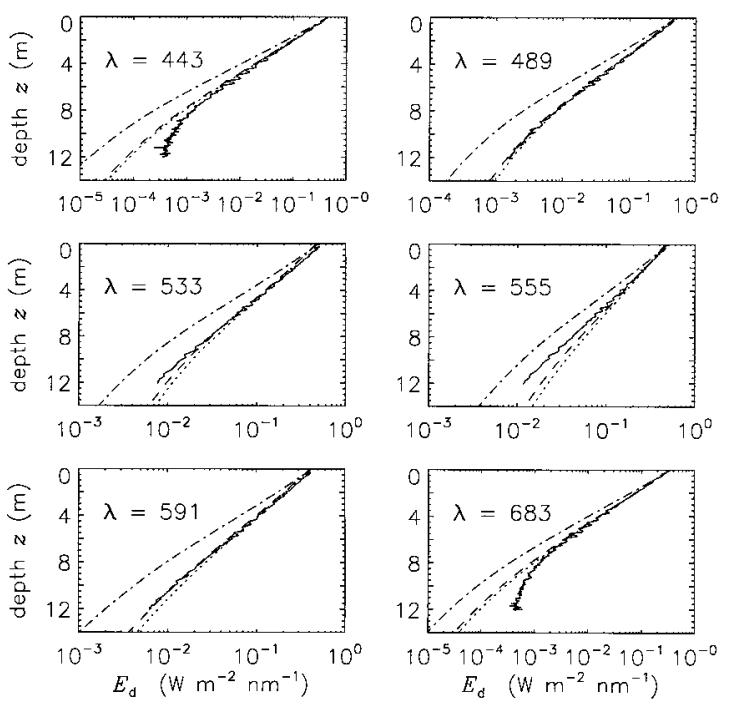

Fig. 14. Comparison of measured and HYDROLIGHT-predicted downwelling plane irradiances at selected wavelengths. Solid curve, measured values; dotted curve, predicted values with the FF phase function; dashed curve, predicted values with the measured phase function; dashed-dotted curve, predicted values with the Petzold phase function.

widths were selected to match the nominal $10-\mathrm{nm}$ bandwidths of the OCP instruments (plus intermediate bands between those of the instruments); band centers were at 412-694 $\mathrm{nm}$. We ran HYDROLIGHT to a depth of $17 \mathrm{~m}$ using the measured IOP profiles; below $17 \mathrm{~m}$ the water was assumed to be infinitely deep and homogeneous, with IOPs equal to those at $17 \mathrm{~m}$.

Three HYDROLIGHT runs were made, each with a different way to determine the particle phase function. First, HYDROLIGHT used the depth- and wavelength-dependent particle backscatter fraction seen in Fig. 12 to determine a FF phase function with the given $B_{p}$ at each depth and wavelength, as described in Section 4. For wavelengths outside the range of the measured $b_{p}$ or $b_{b p}$ values, values at the nearest wavelength were used to determine $B_{p}$. Linear interpolation in depth and wavelength was used in between the measured values. Second, we ran HYDROLIGHT using the measured phase function of Fig. 13 at each depth and wavelength. Third, we ran HYDROLIGHT using the Petzold phase function at each depth and wavelength. In these three runs all inputs except the particle phase functions were identical.

Figure 14 shows the computed downwelling irradiances $E_{d}$ for six wavelengths corresponding to the OCP radiometers. In each panel, the solid curve is the measured data, the dotted curve is the HYDROLIGHT output with depth- and wavelength-dependent FF phase functions, the dashed curve is HYDROLIGHT with the measured phase function, and the dashed-dotted curve is HYDROLIGHT with the Petzold phase function. Measurements were made to a depth of $12 \mathrm{~m}$. At 443 and $683 \mathrm{~nm}$ there was insufficient light for trustworthy $E_{d}$ measurements below $\sim 6 \mathrm{~m}$ because of the high 

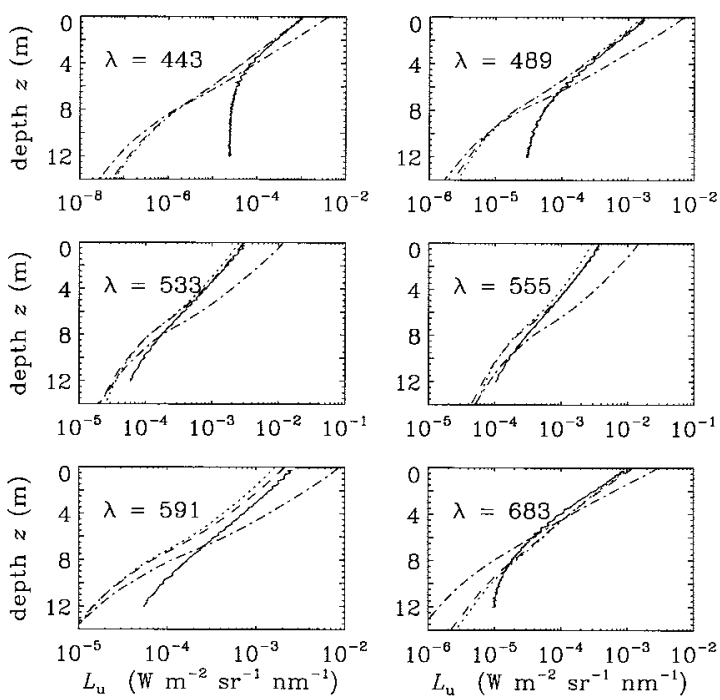

Fig. 15. Comparison of measured and HYDROLIGHT-predicted upwelling radiances at selected wavelengths. Solid curve, measured values; dotted curve, predicted values with the $\mathrm{FF}$ phase function; dashed curve, predicted values with the measured phase function; dashed-dotted curve, predicted values with the Petzold phase function.

absorption by CDOM at $443 \mathrm{~nm}$ and by water at 683 $\mathrm{nm}$; a roll-off of the measurements can be seen as the signal approaches the instrument noise level. (Wavelength $412 \mathrm{~nm}$ showed an even faster roll-off with depth and is therefore omitted from Fig. 14.) In all cases the $\mathrm{FF}$ and measured phase functions give much better agreement with the measured $E_{d}$ than does the Petzold phase function, whose $E_{d}$ values decay too quickly with depth. In these runs the diffuse attenuation depth $1 / K_{d}$ reached a maximum of $\sim 4 \mathrm{~m}$ near $570 \mathrm{~nm}$; thus $90 \%$ of the water-leaving radiance comes from the upper $4 \mathrm{~m}$ of the water column. ${ }^{33}$ For a given depth and wavelength, we define the percentage error between model and data values as 100 (model data)/data; a rms percentage error is then defined from the percentage errors at the six wavelengths. At a depth of $4 \mathrm{~m}$, the computed $E_{d}$ values were within $14 \%$ of the measured values at all wavelengths when we used the FF phase function; the rms error (over the six wavelengths) was $10 \%$. The maximum error when we used the measured phase function was $19 \%$ (rms, 13\%). When we used the Petzold phase function, the computed errors were as much at $-54 \%$ (i.e., the measured $E_{d}$ were over twice the predicted values; $\mathrm{rms}, 45 \%$ ). At $12 \mathrm{~m}$, the $\mathrm{FF}$ values were $69 \%$ too large at $555 \mathrm{~nm}$ but were within $27 \%$ of the measured $E_{d}$ at 489,533 , and $591 \mathrm{~nm}$. The values computed with the measured phase function were within $46 \%$ at wavelengths between 489 and 591. The Petzold values were as much as a factor of 4.8 too low (a $-79 \%$ error). The FF and measured phase function $E_{d}$ values had a rms difference of $17 \%$ at $12 \mathrm{~m}$, with the $\mathrm{FF}$ values being higher in all cases.

Figure 15 shows the corresponding results for $L_{u}$. The roll-off in $L_{u}$ at depth for some wavelengths is

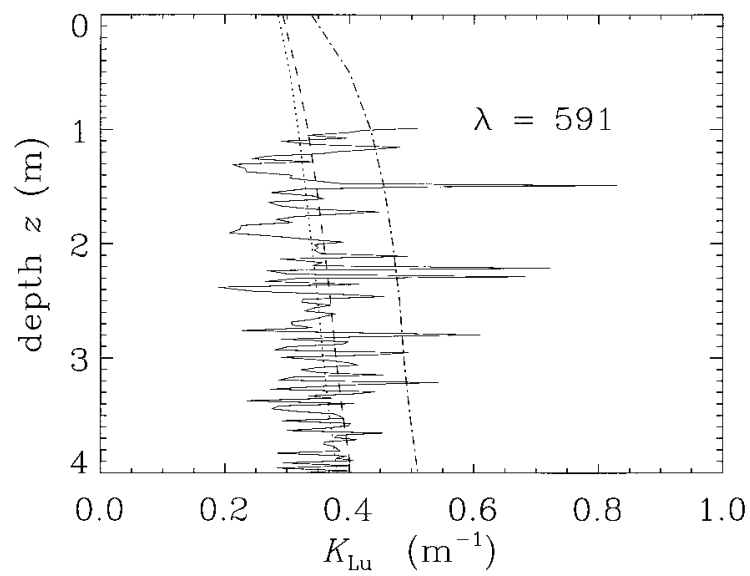

Fig. 16. Comparison of measured and HYDROLIGHT-predicted diffuse attenuation for upwelling radiance at $591 \mathrm{~nm}$. Solid curve, measured values; dotted curve, predicted values with the FF phase function; dashed curve, predicted values with the measured phase function; dashed-dotted curve, predicted values with the Petzold phase function.

due to insufficient light for the $L_{u}$ instrument; this instrument sensitivity was not an issue in the upper few meters of the water. At $0.5-\mathrm{m}$ depth, HYDROLIGHT values predicted with the $\mathrm{FF}$ phase function show a rms error of $31 \%$, with a maximum error of $-43 \%$ at $591 \mathrm{~nm} . \quad L_{u}(0.5-\mathrm{m})$ values predicted with the measured phase function had a rms error of $19 \%$ (maximum error of $-28 \%$ at $591 \mathrm{~nm}$ ). However, the values predicted with the Petzold phase function had a rms error of $183 \%$ (i.e., they were a factor of 2.8 times as large) with a maximum error of $218 \%$ at 555 $\mathrm{nm}$. Use of a phase function with the correct backscatter fraction thus reduced rms percentage errors in the predicted $L_{u}$ just beneath the sea surface by roughly an order of magnitude.

The comparisons in Figs. 14 and 15 were between measured and predicted radiometric magnitudes. They thus include effects of imperfect instrument calibration as well as possible modeling errors. That is to say, for example, the difference in the measured and predicted $L_{u}$ values at $591 \mathrm{~nm}$ may have been due to an inaccurate $L_{u}$ measurement, or to inaccurate input to HYDROLIGHT. We can remove this type of instrument calibration effect by comparing measured and computed apparent optical properties, such as diffuse attenuation functions or the remote-sensing reflectance.

Figure 16 shows the diffuse attenuation function for upwelling radiance $K_{L_{u}}$ at $591 \mathrm{~nm}$, the wavelength that gave the poorest comparison of $L_{u}$ magnitudes just beneath the surface, as was seen in Fig. 15. It is difficult to compute $K_{L}=-\mathrm{d}\left[\ln \left(L_{u}(z)\right] / \mathrm{d} z\right.$ with finite differences because of noise in the measured $L_{u}$ profiles (possibly due to wave focusing ${ }^{34}$ ), which is barely noticeable on the plot scales of Fig. 15. The measured data plotted in Figs. 14 and 15 consist of 594 depth values as acquired during the hydrocast. We smoothed these data with a 51-point boxcar filter (a width corresponding to $\sim 1 \mathrm{~m}$ of depth) before finite 


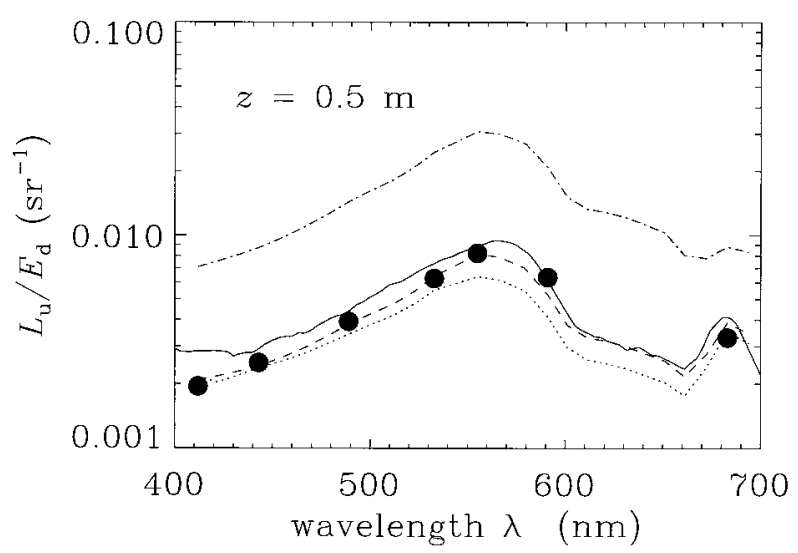

Fig. 17. Comparison of measured and HYDROLIGHT-predicted remote-sensing reflectance at $0.5-\mathrm{m}$ depth. Solid curve, measured by Hyper-TSRB [as corrected by Eq. (10)]; filled circles, measured values by in-water OCP; dotted curve, predicted values by HYDROLIGHT with the FF phase function; dashed curve, predicted values with the measured phase function; dashed-dotted curve, predicted values with the Petzold phase function.

differencing to obtain the data $K_{L_{u}}$ profile seen in Fig. 16. With this smoothing, the computed profile had a rms noise level that was $30 \%$ of the signal. It is clear that the HYDROLIGHT-computed $K_{L_{u}}$ are in good agreement with the measured values when we use either the FF or the measured phase function, but that the Petzold phase function gives a $K_{L}$ that is significantly larger than measured. The other wavelengths show similar results.

Figure 17 shows another comparison of predicted and computed apparent optical properties. The filled circles show the measured in-water remotesensing reflectance $R_{\mathrm{rs}}(z, \lambda)=L_{u}(z, \lambda) / E_{d}(z, \lambda)$ at a depth of $0.5 \mathrm{~m}$ for the wavelengths of the in-water OCP instrument. The solid curve shows the same quantity as measured by the Hyper-TSRB. The Hyper-TSRB, which is supported by a surface buoy, measures $E_{d}$ in air and $L_{u}$ at a depth of $0.6 \mathrm{~m}$. We transformed these measured values to a depth of $0.5 \mathrm{~m}$ using the HYDROLIGHT output from the run with the FF phase function as follows:

$$
\frac{L_{u}(0.5 \mathrm{~m})}{E_{d}(0.5 \mathrm{~m})}=\frac{L_{u}{ }^{T}(0.6 \mathrm{~m})}{E_{d}{ }^{T}(\text { air })} \frac{E_{d}{ }^{H}(\text { air })}{E_{d}{ }^{H}(0.5 \mathrm{~m})} \frac{L_{u}{ }^{H}(0.5 \mathrm{~m})}{L_{u}{ }^{H}(0.6 \mathrm{~m})} .
$$

Here the superscript $T$ denotes values measured by the Hyper-TSRB, and the superscript $H$ denotes values computed by HYDROLIGHT. The HYDROLIGHTcomputed factors in Eq. (10) essentially move the Hyper-TSRB $E_{d}$ from the air to a $0.5-\mathrm{m}$ depth and move the Hyper-TSRB $L_{u}$ from 0.6 to $0.5 \mathrm{~m}$. Neither the Hyper-TSRB nor the OCP measurements were corrected for instrument self-shading effects on the $L_{u}$ values, although self-shading can be significant in some situations. ${ }^{35,36}$ The dotted curve in Fig. 17 is the $L_{u} / E_{d}$ ratio at $0.5 \mathrm{~m}$ as computed by HYDROLIGHT with the FF phase function; the dashed curve is the ratio computed with the measured phase function, and the dashed-dotted curve is the HYDROLIGHT output with the Petzold phase function.

The OCP and Hyper-TSRB measurements differ by a rms error of $22 \%$ (computed for the seven OCP wavelengths), with the greatest difference (46\%) being at $412 \mathrm{~nm}$. This error is larger than the rms errors between OCP and HYDROLIGHT with FF, $18 \%$, and between OCP and HYDROLIGHT with the measured phase function, $10 \%$. The rms error between the OCP measurements and HYDROLIGHT with the Petzold phase function is $230 \%$. Again, we can see that use of a phase function having the proper backscatter fraction reduces the percentage error in the computed remote-sensing reflectance by an order of magnitude. The differences in predictions and data are then comparable to the differences in data as measured by independent instruments. Note that these near-surface measurements are influenced by the IOPs in the upper few meters of the water column; thus the depth dependence of $B_{p}$ below $z \approx 7 \mathrm{~m}$, as in Fig. 12, has little effect on the simulations in Fig. 17.

HYDROLIGHT predicted the chlorophyll fluorescence peak at $683 \mathrm{~nm}$ fairly well when we used its default chlorophyll fluorescence quantum efficiency of 0.02 . Note also that the fluorescence peak is higher compared with $R_{\mathrm{rs}}$ near $660 \mathrm{~nm}$ for the $\mathrm{FF}$ and measured phase functions than for Petzold. This is because isotropically emitted fluorescence contributes relatively more to the upwelling radiance in the lowbackscatter cases than it does for the highbackscatter Petzold phase function.

\section{Conclusions}

HYDROLIGHT simulations show that, for a given backscatter fraction, the shape of the total phase function at intermediate- ( $\sim 5-90 \mathrm{deg})$ and backward- (90-180 deg) scattering angles can have a significant effect on computed underwater radiances, irradiances, and reflectances (Figs. 3-6). The relationship between the phase function shape and the light field is not simple because the solar angle and multiple scattering determine the contributions of each scattering angle to the total light field. However, the exact shape of the phase function in backscatter directions does not greatly affect the light field, so long as the overall shape of the phase function does not deviate greatly from the correct shape (comparison of Petzold, FF, flat back, and OTHG in Figs. 3-6). It is crucial that the phase function have the correct backscatter fraction (Figs. 8-10). Depending on the nature and concentrations of microbial and mineral particles in the water, particle backscatter fractions in the ocean can vary from a fraction of a percent to several percent. The physically based FF phase function can be used to generate realistic analytic phase functions having any desired backscatter fraction (Fig. 7). In particular, the FF phase function can be used to generate depth- and wavelength-dependent phase functions whose backscatter fractions agree with measured values. In an example analysis of case II water, use 
of a depth- and wavelength-dependent FF phase function for the particle component gave much better agreement with measured downwelling irradiances and upwelling radiances than did a commonly used Petzold phase function, which had too large a backscatter fraction (Figs. 14-17). The predictions obtained with a FF phase function were in good agreement with those obtained with a measured phase function. These simulations show that use of the correct particle phase function is just as necessary for accurate prediction of underwater light fields as is use of the correct absorption and scattering coefficients. If the particle phase function is not measured, a FF phase function as determined by measured backscatter fractions can provide a satisfactory substitute.

This research was funded in part by HYDROLIGHT revenues and in part by the Environmental Optics Program of the U.S. Office of Naval Research. The Hyperspectral Coastal Ocean Dynamics Experiment data used in Section 6 were provided by S. Pegau of Oregon State University and M. Lee, M. Shibonov and G. Korotaev of the Marine Hydrophysical Institute of Sebastopol, Ukraine.

\section{References}

1. C. D. Mobley and L. K. Sundman, Hydrolight 4.1 Users' Guide (Sequoia Scientific, Inc., Redmond, Wash., 2000); see also www.sequoiasci.com.

2. C. D. Mobley and L. K. Sundman, Hydrolight 4.1 Technical Documentation (Sequoia Scientific, Inc., Redmond, Wash., 2000).

3. T. J. Petzold, "Volume scattering functions for selected ocean waters," Tech. Rep. SIO 72-78 (Scripps Institution of Oceanography, San Diego, Calif., 1972).

4. C. D. Mobley, B. Gentili, H. R. Gordon, Z. Jin, G. W. Kattawar, A. Morel, P. Reinersman, K. Stamnes, and R. H. Stavn, "Comparison of numerical models for computing underwater light fields," Appl. Opt. 32, 7484-7504 (1993).

5. H. R. Gordon, "Modeling and simulating radiative transfer in the ocean," in Ocean Optics, R. W. Spinrad, K. L. Carder, and M. J. Perry, ed. (Oxford U. Press, Oxford, UK, 1994), pp. 1-39.

6. G. N. Plass, G. W. Kattawar, and T. J. Humphreys, "Influence of the oceanic scattering phase function on the radiance," J. Geophys. Res. 90(C2), 3347-3351 (1985).

7. H. R. Gordon, "Sensitivity of radiative transfer to small-angle scattering in the ocean: quantitative assessment," Appl. Opt. 32, 7505-7511 (1993).

8. C. D. Mobley, Light and Water: Radiative Transfer in Natural Waters (Academic, San Diego, Calif., 1994).

9. G. Fournier and J. L. Forand, "Analytic phase function for ocean water," in Ocean Optics XII, J. S. Jaffe, ed., Proc. SPIE 2258, 194-201 (1994).

10. G. Fournier and M. Jonasz, "Computer-based underwater imaging analysis," in Airborne and In-water Underwater Imaging, G. Gilbert, ed., Proc. SPIE 3761, 62-77 (1999).

11. G. Fournier, Defense Research Establishment Valcartier, ValBelair, G3J 1X5, Canada (personal communication, 2000).

12. L. C. Henyey and J. L. Greenstein, "Diffuse radiation in the galaxy," Astrophys. J. 93, 70-83 (1941).

13. G. W. Kattawar, "A three-parameter analytic phase function for multiple scattering calculations," J. Quant. Spectrosc. Radiat. Transfer 15, 839-849 (1975).

14. V. I. Haltrin, "Two-term Henyey-Greenstein light scattering phase function for seawater," in IGARSS '99: Proceedings of the International Geoscience and Remote Sensing Symposium (Institute of Electrical and Electronics Engineers, New York, 1999), pp. 1423-1425.

15. A. W. Harrison and C. A. Coombes, "An opaque cloud cover model of sky short wavelength radiance," Sol. Energy 41, 387392 (1988).

16. W. W. Gregg and K. L. Carder, "A simple spectral solar irradiance model for cloudless maritime atmospheres," Limnol. Oceanogr. 35, 1657-1675 (1990).

17. W. S. Pegau, J. S. Cleveland, W. Doss, C. D. Kennedy, R. A. Maffione, J. L. Mueller, R. Stone, C. C. Trees, A. D. Weidemann, W. H. Wells, and J. R. V. Zaneveld, "A comparison of methods for the measurement of the absorption coefficient in natural waters," J. Geophys. Res. 100, 13201-13220 (1995); see also www.wetlabs.com.

18. R. A. Maffione and D. R. Dana, "Instruments and methods for measuring the backward-scattering coefficient of ocean waters," Appl. Opt. 36, 6057-6067 (1997); see also www.hobilabs.com.

19. C. Moore, M. S. Twardowski, and J. R. V. Zaneveld, "The ECO VSF-a sensor for determination of the volume scattering function in the backward direction," in Ocean Optics XV, Proceedings on CD (U.S. Office of Naval Research, Ocean, Atmospheric and Space Science and Technology Department, Arlington, Va., 2000); see also www.wetlabs.com.

20. M. S. Twardowski, E. Boss, J. B. Macdonald, W. S. Pegau, A. H. Barnard, and J. R. V. Zaneveld, "A model for estimating bulk refractive index from the optical backscattering ratio and the implications for understanding particle composition in Case I and Case II waters," J. Geophys. Res. 106(C7), 14129-14142 (2001).

21. O. Ulloa, S. Sathyendranath, and T. Platt, "Effect of the particle-size distribution on the backscattering ratio in seawater," Appl. Opt. 33, 7070-7077 (1994).

22. D. Stramski, A. Bricaud, and A. Morel, "A database of singleparticle optical properties," in Ocean Optics XIV, Proceedings on CD (U.S. Office of Naval Research, Ocean, Atmospheric and Space Science and Technology Department, Arlington, Va., 1998).

23. D. Stramski, A. Bricaud, and A. Morel, "Modeling the inherent optical properties of the ocean based on the detailed composition of the planktonic community," Appl. Opt. 40, 2929-2945 (2001).

24. M. Lee and M. R. Lewis, "Measurement of the optical volume scattering function in the ocean," J. Atmos. Oceanic Technol., submitted for publication.

25. M. S. Twardowski, J. M. Sullivan, P. L. Donaghay, and J. R. V. Zaneveld, "Microscale quantification of the absorption by dissolved and particulate material in coastal waters with an ac9," J. Atmos. Oceanic Technol. 16, 691-707 (1999).

26. J. R. V. Zaneveld, J. C. Kitchen, and C. M. Moore, "The scattering error correction of reflecting-tube absorption meters," in Ocean Optics XI, G. Gilbert, ed., Proc. SPIE 1750, 187-200 (1994).

27. C. S. Roesler, Bigelow Laboratory Ocean Sciences, Boothbay Harbor, Maine 04573 (personal communication, 2001).

28. A. Morel and S. Maritorena, "Bio-optical properties of oceanic waters: a reappraisal," J. Geophys. Res. 106, 7163-7180 (2001).

29. H. R. Gordon and A. Morel, Remote Assessment of Ocean Color for Interpretation of Satellite Visible Imagery, a Review; Lecture Notes on Coastal and Estuarine Studies (Springer-Verlag, New York, 1983), Vol. 4.

30. M. J. Perry, Darling Marine Center, University of Maine, Walpole, Maine 04573 (personal communication, 2001).

31. R. M. Pope and E. S. Fry, “Absorption spectrum (380-700 nm) 
of pure water. II. Integrating cavity measurements," Appl. Opt. 36, 8710-8723 (1997).

32. A. Morel, "Optical properties of pure water and pure seawater," in Optical Aspects of Oceanography, N. G. Jerlov and E. S. Nielsen, eds. (Academic, New York, 1974), pp. 1-24.

33. H. R. Gordon and W. R. McCluney, "Estimation of the depth of sunlight penetration in the sea for remote sensing," Appl. Opt. 14, 413-416 (1975).

34. J. R. V. Zaneveld, E. Boss, and A. Barnard, "Influence of sur- face waves on measured and modeled irradiance profiles," Appl. Opt. 40, 1442-1449 (2001).

35. H. R. Gordon and K. Ding, "Self-shading of in-water optical instruments," Limnol. Oceanogr. 37, 491-500 (1992).

36. R. A. Leathers, T. V. Downes, and C. D. Mobley, "Selfshading correction for upwelling sea-surface radiance measurements made with buoyed instruments," Opt. Exp. 8, 561-570 (2001); http://www.opticsexpress.org/oearchive/ source/32933.htm. 